\title{
Recht und Kultur
}

- Das Subjekt im Spiegel der Institution: Pierre Legendres dogmatische Anthropologie -

\section{Summary}

The existing contribution pursues a synopsis of law and culture based on the concept of the institution. Pierre Legendre's dogmatic anthropology provides a relevant theoretical reference that defines the human being as an image-bound entity based on alterity whose subjectivity is constituted by the entry into the institution. Against the background of the Freudian hypothesis of an analogy between individual and cultural genesis, the law becomes recognisable as the central mediator of a fictitious foundation of power, which constitutes the core of the cultural identity.

\section{Résumé}

Cet article présente une vision synoptique du droit et de la culture à travers la notion d'institution. Il s'inspire principalement de la théorie de l'anthropologie dogmatique de Pierre Legendre, qui définit l'homme comme un être lié à l'image et fondé sur l'altérité, dont la subjectivité se construit lorsqu'il intègre l'institution. Avec en toile de fond l'hypothèse freudienne d'une analogie entre la genèse individuelle et culturelle, le droit est défini comme le principal vecteur d'un fondement fictif du pouvoir, qui constitue le cour de l'identité culturelle.

„Lassen Sie sich nicht beirren durch die Oberflächen; in den Tiefen wird alles Gesetz."

Rainer Maria Rilke

I. Eine Zusammenschau der Begriffe Recht und Kultur leuchtet zunächst insofern ein, als das Recht impliziter und vielleicht ja sogar konstitutiver Bestandteil von Kultur ist. Jede Kultur basiert auf einem System normativer Grundsätze, die das Zusammenleben der in dieser Kultur beheimateten Subjekte mehr oder weniger verbindlich regelt. Fasst man Kultur als ein spezifisches Setting erwartbarer Verhaltensmuster, d.h. als eine Strategie, durch Antizipation des Zukünftigen Kontingenz zumindest auf der Ebene der personenbezogenen Interaktion zu reduzieren, so wird deutlich, dass ein derartiges kulturelles Setting selbst wiederum auf einem Ensemble von Geboten und Verboten gründen muss. Umgekehrt kann sich ein solches Ensemble von Geboten und Verboten nur im Rekurs auf die kulturelle Dimension zu einem institutionellen Rechtssystem ausprägen, denn nur mit den Mitteln der Kultur werden Regeln internalisiert, stabilisiert 
und tradiert. Durch Narration, Ritual und Inszenierung wird normatives Wissen nicht nur sichtbar gemacht, sondern auch in einen Modus der Wiederholung gestellt. »Rituale wiederholen das Vergangene im Sinne der szenischen Inkraftsetzung. Sie sind nicht nur Gedenkhandlungen, sondern erheben auch den Anspruch, Vergangenheitsdeutungen durchzusetzen bzw. ein öffentliches Gedächtnis auszubilden. « ${ }^{1}$

Ein spezifisches Problem der Zusammenschau von Recht und Kultur liegt nun darin, dass vor allem der Kulturbegriff so leicht nicht zu definieren ist. Dies liegt zunächst daran, dass >Kultur seit der zweiten Hälfte des 18. Jahrhunderts neben 〉Bildung〈, >Aufklärung « und `Zivilisation ` zu den großen utopischen Leitbegriffen gehört, durch die der Selbstverständigungsprozess einer sich in Progression befindlichen Gesellschaft sichergestellt und strukturiert werden soll. Das Potential dieser Begriffe resultiert zu einem nicht unwesentlichen Teil aus ihrer latenten Überdetermination, weil durch sie Verstehensgrenzen überschritten und Interpretationshorizonte entworfen werden, die erst durch diese Begriffe selbst erschlossen und hervorgebracht wurden. ${ }^{2}$ Reinhart Koselleck hat darauf hingewiesen, dass >Kultur` seit dem 18. Jahrhundert als ein Kollektivsingular gebraucht wird und ohne Genitivattribut auftritt. Diese Ablösung von dem im Genitiv obligat folgenden Objekt der Pflege (etwa: die Kultur des Ackerbaus) ist natürlich die Voraussetzung für die folgenreiche Karriere des Kulturbegriffs. In dieser Weise verselbständigt und von einem konkreten Bezug auf etwas Anderes gelöst, bezeichnet >Kultur` eine Dimension der menschlichen Entwicklung, die sich selbst validiert. $^{3}$

\Kultur rückt damit ein in die Reihe absolut gebrauchter Termini »und wird in diesem Sinne universalisiert wie die Kollektivbegriffe `Geschichte $<$ oder $>$ Natur $<$ - absolut sind sie, weil ihre Extension alles zu umfassen sucht und weil sie damit alle Gegenbegriffe integrieren. Denn was wäre nicht $>$ Natur , was nicht $>$ Geschichte $<-$ und was wäre nicht $>$ Cultur $\ll ? «^{4}$ Tatsächlich umfasst der Kulturbegriff der Kulturwissenschaften mittlerweile alles von den symbolischen Grundlagen des Handelns bis zur Gesamtheit menschlicher Artefakte. Interessant ist ein kurzer Blick in den Katalog einer beliebigen Universitätsbibliothek, Stichwort: >Kulturgeschichte`. Hier finden sich dann meist mehrere tausend Einträge wie: Kulturgeschichte des Wassers, Kulturgeschichte des Geldes, Kulturgeschichte der Beschneidung, Kulturgeschichte des deutschen Bauernhauses, Kulturgeschichte des Strumpfes, Kulturgeschichte des assyrischen Flaschenbildes, Kulturgeschichte der Prostitution, Kulturgeschichte des Spaziergangs, Kulturgeschichte des Fußballs, Kulturgeschichte des Klimas, Kulturgeschichte des Mittelalters, Kulturgeschichte des Salzes usw. usf. Um Goethes Mephisto aus dem ersten Teil des Faust zu

1 Peter Burke, Geschichte als soziales Gedächtnis. In: Aleida Assmann; Dietrich Harth (Hg.), Mnemosyne. Formen und Funktionen der kulturellen Erinnerung. Frankfurt a.M. 1991, S. 289-304, hier S. 293.

2 Vgl. Helmut Peukert, Reflexionen über die Zukunft von Bildung. In: Zeitschrift für Pädagogik 46 (2000), S. 507-524, hier S. 507.

3 Vgl. Niklas Luhmann, Kultur als historischer Begriff. In: Ders., Gesellschaftsstruktur und Semantik. Studien zur Wissenssoziologie der modernen Gesellschaft. Bd. 4. Frankfurt a.M. 1999, S. 31-54, hier S. 40.

4 Jürgen Fohrmann, Das Andere der »Kultur«. Die »Kultur« der Kulturwissenschaft. In: Georg Mein und Markus Rieger-Ladich (Hg.), Soziale Räume und kulturelle Praktiken. Über den strategischen Gebrauch von Medien. Bielefeld 2004, S. 85-100, hier S. 85. 
zitieren: »Auch die Kultur, die alle Welt beleckt, / Hat auf den Teufel sich erstreckt.« (V. 2495 f.)

Die massive Transgression dessen, was der Begriff $>$ Kultur $८$ konzeptionell umfassen soll, ernüchtert freilich. Auf der anderen Seite aber wird gerade dadurch deutlich, was die Fokussierung der Welt im Modus von Kultur leistet, nämlich eben dies: eine spezifische Verdopplung von Welt im Modus von Kultur. Und dies bedeutet konkret eine Umstellung von Beobachtung erster Ordnung auf eine Beobachtung zweiter Ordnung. Kultur eröffnet eine Perspektive für die Beobachtung von Beobachtern. ${ }^{5}$ Dies aber impliziert sofort jene Verdopplung von Welt, wie Niklas Luhmann eindrücklich vor Augen führt:

Kultur ist [...] ein Doppel, sie dupliziert alles, was ist. Daher formuliert sie ein Problem der >Identität $\iota$, das sie für sich nicht lösen kann - und eben deshalb problematisiert. Nach wie vor kann man mit dem Messer schneiden, kann man zu Gott beten, zur See fahren, Verträge schließen oder Gegenstände verzieren. Aber außerdem läßt sich all das ein zweites Mal beobachten und beschreiben, wenn man es als kulturelles Phänomen erfaßt und Vergleichen aussetzt. ${ }^{6}$

Wenn Kultur als Beobachtung zweiter Ordnung einen spezifischen Modus des Vergleichs offeriert, dann zeigt die kulturelle Perspektive zugleich, dass die Welt eben nicht nur so, sondern auch anders sein kann, sie konfrontiert die Wirklichkeit mit einer Alternativversion und stellt auf diese Weise >Weltkontingenz $\prec$ her. ${ }^{7}$ Kultur entsteht, so Luhmann, "wenn der Blick $\mathrm{zu}$ anderen Formen und anderen Möglichkeiten abschweift $\ll,{ }^{8}$ wodurch die Semantik der Kultur alles, was kommuniziert werden kann, mit Kontingenz überzieht und so von jeder Art notwendigem Sinn befreit. ${ }^{9}$ Kultur installiert auf diese Weise eine Praxis des Vergleichens, weil nicht mehr nur die Dinge selbst, sondern fortan eben auch die Art und Weise, wie andere diese Dinge beobachten, beobachtet werden. In diesem Sinne argumentiert dann auch Dirk Baecker, wenn er betont, dass es im Kern um die scheinbar ganz harmlose intellektuelle Geste geht, »irgend etwas für >interessant zu halten und sich mithilfe des Vergleichswissens [...] Gedanken über dieses Interessante zu machen. ${ }^{10}$ Gleichzeitig impliziert dieses Interesse für das Andere und den Anderen zugleich die Einsicht, dass man selbst für diese Anderen ebenfalls zum Objekt des Interesses wird. Wer andere dabei beobachtet, wie sie beobachten, der weiß in der Regel, dass auch diese anderen ihn bei seiner Beobachtung beobachten. Kultur als Praxis des Vergleichens initiiert daher stets auch eine nie zum Ende kommende Reflexion auf sich selbst, die von der Motivation getrieben ist, sich selbst als Anderen zu beobachten, und zwar mit dem Ziel, aus dieser Alteritätserfahrung Profit für das eigene Selbst zu schlagen.

5 Vgl. Niklas Luhmann, Kultur als historischer Begriff, S. 54.

6 Ebd., S. 42.

7 Vgl. dazu auch: Niklas Luhmann, Das Kunstwerk und die Selbstreproduktion der Kunst. In: Hans Ulrich Gumbrecht/K. Ludwig Pfeiffer (Hg.), Stil. Geschichten und Funktionen eines kulturwissenschaftlichen Diskurselements. Frankfurt a.M. 1986, S. 620-672.

8 Niklas Luhmann, Kultur als historischer Begriff, S. 48.

9 Vgl. ebd., S. 51.

10 Dirk Baecker, Wozu Kultur? Berlin 2003, S. 48. 
Der hier unternommene Versuch einer Zusammenschau von Recht und Kultur setzt genau an diesem Punkt an, indem die These vertreten wird, dass Recht und Kultur jene Form von Alterität handhaben, die dem Subjekt als das Fremde im Selbst gegenübertritt. Die zentrale Schaltstelle für diesen Prozess und zugleich der Ort, an dem Recht und Kultur ein unauflösliches Amalgam bilden, ist die Institution. Wir verstehen Institutionen als kulturell unverzichtbare Orte, die die Trennung des Subjekts von sich selbst, d.h. die für den Menschen konstitutive Verbindung zwischen der biologischen und der symbolischen Dimension, garantieren und organisieren. Während dieser Prozess der Menschwerdung in anderen Kulturen von der Religion gewährleistet wird, hat das Abendland diese anthropologische Funktion dem Recht übertragen. ${ }^{11}$ Wir werden im Folgenden dieses spezifische Verständnis des Institutionellen und der Funktion des Rechts erläutern.

II. Einigkeit besteht darin, dass der verstehende Zugriff auf die Wirklichkeit das Ergebnis einer Differenzierungsleistung ist. Draw a distinction!, ${ }^{12}$ lautet die markige Aufforderung von George Spencer Brown. Um die Wirklichkeit zu beobachten, muss sie als von mir unterschieden angenommen werden, und diese Differenz ist auch für den reflexiven Selbstbezug konstitutiv. Man tritt nicht als biologisches Fleisch in die symbolische Ordnung ein, sondern als beschriebener Köper, d.h. als domestiziertes, kulturell zugerichtetes Artefakt, als ein Bild, mit dem man sich zu identifizieren gelernt hat und das einem doch als ein von sich entfremdetes entgegentritt. »Je est un autre«, sagt Arthur Rimbaud. ${ }^{13}$ Alterität erweist sich so als eine Bezugsgröße, die das Ich im Prozess seiner Selbstkonstitution sich selbst gegenüber erfährt. ${ }^{14}$ Der Mythos des Narziss ist dafür nur das anschaulichste Beispiel. Hier begegnet auch erstmalig die Metapher des Spiegels, denn Narziss betrachtet ja sein Spiegelbild, das ihm aus dem Wasser entgegenblickt und das er - zunächst - nicht auf sich selbst bezieht. In den Metamorphosen des Ovid heißt es:

Und während er den Durst zu stillen trachtete, wuchs in ihm ein anderer Durst. Während er trinkt, erblickt er das Spiegelbild seiner Schönheit, wird von ihr hingerissen, liebt eine körperlose Hoffnung, hält das für einen Körper, was nur Welle ist. $^{15}$

Narziss ist verzweifelt, dass er ein »nichtiges Spiegelbilde«, sein Gesicht selbst, nicht zu erreichen vermag. Ovid schreibt in den Metamorphosen: »O utinam a nostro secedere corpore possem!« Wörtlich übersetzt: 〉O könnte ich mich doch von unserem Körper lösen!^ Es heißt nicht meinem Körper, sondern unserem Körper. Narziss wendet sich an

11 Vgl. dazu Alain Supiot, Homo juridicus. Essais sur la fonction anthropologique du Droit. Paris, Seuil 2005.

12 Georges Spencer Brown, Laws of Form. London 1969, S. 3.

13 Arthur Rimbaud, Brief an Paul Demeny, 15. Mai 1871. In: Correspondance inédite (1870 -1875) d'Arthur Rimbaud, précédée d'une introduction de Roger Gilbert-Lecomte. Paris 1929, S. 49-61, hier S. 51.

14 Vgl. Georg Mein, Choreografien des Selbst. Studien zur institutionellen Dimension von Literalität. Wien, Berlin 2011, S. 23 ff.

15 P. Ovidius Naso, Metamorphosen III, S. 416-419. Übers. und hg. v. Michael von Albrecht. Stuttgart, Reclam 1994, S. 153. 
sein Spiegelbild, als sei es ein anderer, unterstellt aber, dass er mit diesem Bild denselben Körper teilt. Damit unterstellt er zugleich ein unauflösliches Band zwischen Körper und Bild. Diese Szene verdeutlicht, dass der Körper dem Subjekt durch das Bild verliehen wird, d.h. dass erst durch die Spiegelszene sich der Körper vom Status eines biologischen Objekts löst und den einer Fiktion annimmt. Hier handelt es sich somit um einen Trennungsvorgang, durch den das menschliche Erkennen in ein entscheidendes Stadium gelenkt wird: »Als Ursprungspunkt seines eigenen Daseins in der Welt entdeckt sich das Ich nur über dieses andere Objekt, sein Bild. « ${ }^{16}$

Freilich kann man diese Geschichte auch noch einmal anders erzählen, etwa mit Blick auf die je individuelle Konstitution des Selbst im frühkindlichen Stadium. Hier kommt der bekannte Text des Psychoanalytikers Jacques Lacan ins Spiel: Das Spiegelstadium als Bildner der Ich-Funktion. Das in den Spiegel schauende Kind erblickt zwischen dem sechsten und dem achtzehnten Lebensmonat ein imaginäres Bild von der Gestalt seines Körpers, dessen somatische Einheit in signifikantem Gegensatz zum Stadium der eigenen körperlichen Wahrnehmung steht. Das Kind sieht seinen ganzen Körper im Spiegel, den es zu seinem derzeitigen Entwicklungsstand überhaupt nicht in dieser Ganzheit wahrnehmen kann. Dem Bild dieser Ganzheit entspricht somit keine subjektive Erfahrung; vielmehr soll der bloße Anblick der vollständigen Form dem Subjekt eine imaginäre Beherrschung seines Körpers ermöglichen, die gegenüber der realen Beherrschung verfrüht ist. ${ }^{17} \mathrm{In}$ >jubilatorischer Geschäftigkeit« schafft sich das Kind, das noch eingetaucht ist in motorische Ohnmacht, kraft seines Blicks ein Ideal-Ich. Anders formuliert, das real völlig hilflose Kind begrüßt im Spiegel seine künftige körperliche Einheit. Dieses durch das Spiegelbild entworfene Ideal-Ich bezeichnet Lacan als moi und kennzeichnet seine Grundstruktur als narzisstisch. So versuche das moi alle störenden Außeneinflüsse von seiner Selbstliebe fernzuhalten, sowie alle Objektbeziehungen Ichzentriert zu gestalten. Folgerichtig wird aus dieser Perspektive das andere nur als Projektionsfläche des eigenen Ich gesehen:

Es ist das Bild seines Körpers, das das Prinzip jeder Einheit ist, die er an Objekten wahrnimmt. Von diesem Bild nimmt er die Einheit nur außerhalb und in einer antizipierenden Art und Weise wahr. Aufgrund dieser doppelten Beziehung, die er zu sich selbst hat, werden sich sämtliche Objekte seiner Welt immer um den irrenden Schatten seines eigenen Ich strukturieren. Sie werden alle einen fundamental anthropomorphen, wir sollen sogar sagen egomorphen Charakter haben. ${ }^{18}$

Insofern kann man sagen, dass das imaginäre Bild im Spiegel das Subjekt wie in einem Bann gefangen hält, denn das Spiegelstadium richtet eine spezifische Beziehung zwischen dem Organismus und seiner Realität ein, die unter dem Signum einer Imago steht, »kraft der das Subjekt in einer Fata Morgana die Reifung seiner Macht vorweg-

16 Pierre Legendre, Gott im Spiegel. Untersuchung zur Institution der Bilder. Aus dem Französischen von Sabine Hackbarth und Verena Reiner. Wien, Berlin 2011, S. 68.

17 Vgl. Jacques Lacan, Freuds technische Schriften (= Das Seminar. Buch I). Weinheim/Berlin 1990, S. 105.

18 Jacques Lacan, Das Ich in der Theorie Freuds und in der Technik der Psychoanalyse (Das Seminar. Buch II). Weinheim, Berlin 1991, S. 213. 
nimmt «. ${ }^{19}$ Daher lässt sich das Spiegelstadium als eine Identifikation verstehen, und zwar im vollen Sinne, »den die Psychoanalyse diesem Terminus gibt«, wie Lacan betont, nämlich »als eine beim Subjekt durch die Aufnahme eines Bildes ausgelöste Verwandlung. ${ }^{20}$ In seinem Seminar über Freuds technische Schriften bezeichnet Lacan das Spiegelstadium als ein »ursprüngliche[s] Abenteuer«. Hier mache der Mensch zum ersten Mal die Erfahrung, »daß er sich sieht, sich reflektiert und sich als anders begreift als er ist - die wesentliche Dimension des Menschlichen, die sein ganzes Phantasieleben strukturiert. ${ }^{21}$ Aufgrund dieser Erfahrung von Identität im Fremden, im Anderen des Selbst erweise sich die Subjektkonstitution als Moment, in dem der kindliche Mensch einen grundlegenden und lebenslang anhaltenden Mangel erfahre: Im Blick im Spiegel erlebt das Individuum eine Entfremdung zwischen dem, was es wirklich ist und spürt, und dem, was es sieht und als das >Ich< erkennt.

Der entscheidende Punkt in Lacans Ausführungen über das Spiegelstadium ist nun, dass nur durch die Einschaltung eines Dritten, der die Trennung zwischen Spiegelbild und Subjekt authentifiziert, die Entfremdung erträglich und die Subjektkonstitution erfolgreich vollzogen wird. Am Rand des Spiegelstadiums gewinnt diese dritte Person an Bedeutung, wenn es um »jenes Tauschen von Blicken« geht, welches sich darin manifestiert, »daß das Kind sich dem zuwendet, der ihm auf irgend eine Weise assistiert. $\ll^{22}$

Dieser Dritte, der die Trennung zwischen Subjekt und Bild zugleich bestätigt wie überbrückt, spielt nun auch jenseits der Psychogenese eine Rolle, und zwar genau dann, wenn man der These Sigmund Freuds folgt, derzufolge »die Kulturentwicklung [...] weitgehende Ähnlichkeit mit der des Einzelnen hat und mit denselben Mitteln arbeitet. $\ll^{23}$ Mit anderen Worten, die narzisstische Ausrichtung des Subjekts dominiert auch die Konstitution von Kultur, die ebenfalls von einem Bildverhältnis geprägt ist, und zwar im spiegelnden Blick des Anderen. Bernhard Waldenfels hat die Tatsache, dass sich das Subjekt nicht aus sich selbst heraus setzen kann, sondern einer dritten Dimension bedarf, um gegründet zu werden, wie folgt formuliert: »Was wir sind, sind wir [...] nicht auf Grund individueller oder gemeinsamer Entwürfe, sondern wir werden überhaupt erst zu einem Wir durch den beherrschenden Blick des Dritten, der sich im Extremfalle dem göttlichen Blick eines >absoluten Dritten` annähert. « ${ }^{24}$ Genau dies ist der

19 Jacques Lacan, Das Spiegelstadium als Bildner der Ichfunktion. In: Ders., Schriften I. Hg. v. Norbert Haas. 3., korr. Aufl. Weinheim, Berlin 1991, S. 61-70, hier: S. 64.

20 Ebd.

21 Jacques Lacan, Freuds technische Schriften (= Das Seminar. Buch I). Weinheim/Berlin 1990, S. 105.

22 Jacques Lacan, Von dem, was uns vorausging. In: Ders., Schriften III. Hg. v. Norbert Haas. 3., korr. Aufl. Weinheim, Berlin 1994, S. 7-14, hier S. 13.

23 Sigmund Freud, Das Unbehagen in der Kultur. In: Studienausgabe. Hg. v. Alexander Mitscherlich u.a., Frankfurt a.M. 2000, Bd. IX, S. 269.

24 Bernhard Waldenfels, Topographien des Fremden. Frankfurt a.M. 2008, S. 115. 
Punkt, an dem die Institution ins Spiel kommt - ihr fällt die Funktion jenes trennenden und vermittelnden Dritten auf der Ebene der Kultur zu. ${ }^{25}$

III. Institutionen können zunächst als durch Tradition und Gesetz legitimierte Organisationseinheiten begriffen. Ihre Funktion ist es, durch Normierung von Handlungsabläufen gewisse Verbindlichkeiten zu schaffen, durch die die Subjekte entlastet werden. Durch institutionelle Normierung lässt sich das Verhalten anderer antizipieren und so Kontingenz reduzieren. Gleichzeitig aber treten Institutionen als eine fremde Macht auf, sie legen den Angehörigen einer Kultur gewisse Verhaltensregeln auf, die diesen mitunter nicht intuitiv einleuchten, aber selten hinterfragt werden. »Der Glanz, der sie umgibt, überstrahlt ihre Fragwürdigkeit. $«^{26}$

Die Idee des Institutionellen lässt sich jedoch noch sehr viel basaler denken - und eben dies ist der Ansatz, den Pierre Legendre in seiner von ihm so benannten Dogmatischen Anthropologie verfolgt. Die römische Formel Vitam instituere aufgreifend, geht Legendre davon aus, dass Institutionen das Leben einrichten. Sie schaffen eine Wirklichkeit, in der Bedeutungszuweisungen überhaupt erst möglich werden und in der das Subjekt einen ihm zugewiesenen Platz findet. Die institutionelle Dimension beschreibt also eine Dimension jenseits der rohen Materie, jenseits der Natur. Und hier könnte man in der Tat den Gegensatz Natur - Kultur anführen, um deutlich zu machen, dass die institutionelle Dimension das Produkt von Zeichenwirkungen ist: Zeichen, die auf etwas anderes verweisen, die etwas repräsentieren. Mit anderen Worten: Zwischen die Wahrnehmung der Dinge und das Subjekt schiebt sich die Repräsentation, eben jene psychische Instanz mit all ihren unbewussten Implikationen, die es nur gibt, weil es Sprache gibt, den Schleier der Worte, der uns von den Dingen trennt. ${ }^{27}$ Der Eintritt des Subjekts in die Sprache ist für Legendre insofern das erste Moment der Institutionalisierung des Lebens.

Legendre identifiziert die Beziehung zwischen Subjekt und Sprache als ein Machtverhältnis, und zwar dergestalt, dass die Sprache das Subjekt beherrscht und nicht umgekehrt. Er bezieht sich dabei unter anderem auf die linguistischen Arbeiten Ferdinand de Saussures, wenn er die »Zuordnung gemäß dem Zeichen, das dem Subjekt zugefügt wird $«,{ }^{28}$ als die $»$ wichtigste der Institutionen $«{ }^{29}$ bezeichnet. Entsprechend bedeute die institutionelle Einrichtung des Lebens somit zunächst und vor allem dies: »die Logik

25 Pierre Legendre bemerkt zu dieser Spiegelszene sehr treffend: »Diese Entdeckung des Selbst und des anderen, des Selbst als anderer - des anderen im Inneren des Selbst - vollzieht sich notwendigerweise über die Präsenz des Dritten, den Spiegel als solchen, nämlich als Fläche, die die Bilder reflektiert, aber auch als Blick und Diskurs, die dem Subjekt vorausgehen und seinen Zugang zur Spiegelszene beherrschen.« Pierre Legendre: Vom Imperativ der Interpretation. In: Georg Mein (Hg.), Die Zivilisation des Interpreten. Studien zum Werk Pierre Legendres. Wien, Berlin 2012, S. 13-26, hier S. 17.

26 Armin Adam/Martin Stingelin, »Vorwort«. In: Dies. (Hg.), Übertragung und Gesetz. Gründungsmythen, Kriegstheater und Unterwerfungstechniken von Institutionen. Berlin 1995, S. 7-12, hier: S. 7.

27 Vgl. Nicole Péruisset-Fache, L'éducation d'homo sapiens: Du savoir à la sagesse? Paris 2007, S. $31 \mathrm{f}$.

28 Pierre Legendre, Das politische Begehren Gottes. Studien über die Montagen des Staates und des Rechts. Aus dem Französischen von Katrin Becker. Wien, Berlin 2012, S. 295 f.

29 Ebd., S. 100. 
des Sprechens ins Werk zu setzen $« .{ }^{30}$ Die Institutionalisierung des Individuums durch den Eintritt in die Sprache komme daher einer zweiten Geburt gleich: »[D]urch Aussprache des Wortes erkennt sich der Sprechende als der Legalität unterworfenes Subjekt an $\ll,{ }^{31}$ einer Legalität, die er als eine Ordnung erkennt, die »ihn und seine Eltern übersteigt $\ll,{ }^{32}$ und in deren institutioneller Struktur er einen ihm spezifisch zugeordneten Platz einnimmt.

Legendre weist hier auf einen Punkt hin, der gerne übersehen wird: Die Sprache ist ein komplexes Regelwerk, dem man sich in gewisser Hinsicht unterwerfen muss, um es zu benutzen. Ihr verführerischer Zauber liegt in der Repräsentation, die trennend und verbindend zugleich ist. Kraft der Sprache müssen die Dinge, über die man sprechen will, nicht mehr real vor Ort sein, will man sich auf sie beziehen - sie müssen noch nicht einmal real existieren. Die Sprache emanzipiert den Menschen von der Wirklichkeit und dies ist die Voraussetzung dafür, diese Wirklichkeit nach den eigenen Wünschen zu organisieren, sie als Lebenswelt einzurichten.

Die Sprache trennt uns von den Dingen, indem sie sie benennt; aber auch unsere Trennung von den Dingen instituiert die Dinge unter einem Namen für das sprechende Subjekt, und sie instituiert daher das Subjekt selbst als Subjekt des sozialen Diskurses der Kategorien, dem sowohl der Name der Dinge angehört als auch die Vernunft dessen, der inmitten der Dinge diese voneinander abteilt. Sich anhand der Sprache von der primären Undurchdringlichkeit loszureißen, den uranfänglichen Schrecken zu bezwingen, einzutreten in den symbolischen Austausch: Die ganze menschliche Unternehmung bringt das institutionelle Prinzip als Gründungsprinzip des Diskurses und des Wortes in den jeweiligen Gesellschaften ins Spiel. ${ }^{33}$

Der entscheidende Punkt ist nun, worauf sich die institutionelle Dimension gründet, woher sie ihre Macht bezieht. Am Beispiel der Sprache und ihrer Repräsentationsfunktion könnte man im Anschluss an Saussures Cours de linguistique générale argumentieren: Die Macht, Bedeutungsverhältnisse zu instituieren, basiert auf Signifikation, d.h. auf der zeicheninternen, >positiven Relation des Signifikanten zu seinem Signifikat. Allerdings sah sich bereits Saussure im Verlauf seiner Untersuchung dazu gezwungen, die rein relationale Natur des linguistischen Systems immer stärker hervorzustreichen, was ihn schließlich zu dem Schluss führte, dass es in der Sprache »nur Verschiedenheiten ohne positive Einzelglieder « gibt. ${ }^{34}$ Diese Einsicht bringt Saussure dazu, den Begriff des sprachlichen Wertes einzuführen, durch den die individuelle Referenz des Zeichens in den Hintergrund tritt und stattdessen seine Position im Verweiszusammenhang aller Zeichen, sein Stellenwert betont wird. Das Zeichen erlangt seine Bestimmtheit somit nur durch den ständigen Abgleich mit allen anderen Zeichen; es zirkuliert im wahrsten Sinne des Wortes wie eine Münze. Die Bedeutung des sprachlichen Werts resultiert aus dem Zueinander-In-Beziehung-Setzen von Signifikanten. Es verhält sich

30 Pierre Legendre, Die Kinder des Textes. Zur Elternfunktion des Staates. Aus dem Französischen von Pierre Mattern. Wien, Berlin, Turia + Kant 2011, S. 40.

31 Pierre Legendre, Das politische Begehren Gottes, S. 63.

32 Pierre Legendre, La Fabrique de l'Homme Occidental, Paris, Fayard 2000, S. 80.

33 Pierre Legendre, Die Kinder des Textes, S. 39.

34 Ferdinand de Saussure, Grundfragen der allgemeinen Sprachwissenschaft. Hg. v. Charles Bally/Albert Sechehaye unter Mitwirk. v. Albert Riedlinger. Übers. v. Herman Lommel. 3. Aufl. Berlin; New York 2010, S. 143. 
hier ähnlich wie beim Warentausch. Auch dort erlangen die Produkte ihren Wert als Waren erst in dem Augenblick, in dem sie auf dem Tauschmarkt zueinander in Beziehung gesetzt werden. Es gibt keinen absoluten Wert, durch den das Produkt >wertig< wird. ${ }^{35}$ Erst die Zirkulation der Waren untereinander generiert ihren Wert. Ebenso ist es bei den sprachlichen Zeichen, die ihren Wert allein durch ihre soziale Zirkulation generieren, denn die physischen Größen der sprachlichen Zeichen, ihre beiden Seiten, Lautbild und Vorstellung, haben selbst keinen positiven Wert, sondern sind innersprachliche Verbindungen mit rein differentiellem Charakter. Spätestens seit Jacques Derridas Saussure-Lektüre ist diese Wertschöpfungstheorie, die Bedeutung generiert, indem sie Zeichen allein auf Zeichen bezieht, zum festen Bestandteil kulturwissenschaftlichen Wissens geworden. Das aber bedeutet, dass im Zentrum dieser Zirkulation nichts ist, das die Repräsentation und die durch sie erzeugte Bedeutung garantiert. Die Mitte ist sozusagen leer.

Legendre, der sich explizit auf Saussure bezieht, erhebt diese konstitutive Leere nun zum Wesensmerkmal des Institutionellen und damit letztlich zum Kern von Subjektivität.

Die Frage: Wer ist nun also das monumentale Subjekt des institutionellen Systems? kann mit dem Selbstbewusstsein des Odysseus beantwortet werden, der zum Zyklopen spricht: Niemand ist mein Name. Diese Äußerung [...] sagt dennoch die Wahrheit: Was wir so gelehrt als die Grundlagen des Rechts bezeichnen, stellt sich zunächst als notwendiges Füllwerk dar, als Inszenierungsdiskurs, der einen leeren Platz authentifizieren soll. Da alle menschlichen Gesellschaften mit dem Sprechen konfrontiert sind, kann keine von ihnen auf diese Arbeit an der Repräsentation verzichten. Um dies zu verstehen, genügt es nicht, zu erkennen, dass hinter dem institutionellen System niemand steht, kein Subjekt aus Fleisch und Blut, sondern es gilt anzuerkennen, dass dieser Niemand ein Name ist. ${ }^{36}$

Explizit thematisiert Legendre die Frage nach dem Grund des Rechts. Diese Frage ist freilich nicht neu - und auch die Antwort, die Legendre vorschlägt, ist zumindest implizit schon lange vor ihm in Erwägung gezogen worden: »Or les loix se maintiennent en crédit, non par ce qu'elles sont justes, mais par ce qu'elles sont loix. C'est le fondement mystique de leur autorité : elles n'en ont point d'autre. $\aleph^{37}$ Die hier von Montaigne formulierte Mystik bzw. Negativität des Fundaments des Rechts ist der abendländischen juristischen Wissenschaft zwar nicht unbekannt, ${ }^{38}$ zugleich aber auch Gegenstand kontinuierlicher Verdrängung. Alain Supiot zufolge ist eben dieser Widerspruch der eigentliche Motor des juristischen Denkens, das den dogmatischen Charakter des Rechts nicht verleugnen kann und zugleich die Wissenschaft zur Quelle des das Recht

35 Vgl. dazu vor allem: Karl Marx, Das Kapital. Kritik der politischen Ökonomie. Bd.1. (MEW 23). Berlin 2008, S. $49 \mathrm{ff}$.

36 Pierre Legendre, Das politische Begehren Gottes, S. 48.

37 Michel de Montaigne, Essais III, 13. »Nun erhalten sich aber die Gesetze in Ansehen, nicht weil sie gerecht sind, sondern weil sie Gesetze sind. Das ist der mystische Grund ihres Ansehens. Einen andern haben sie nicht, worauf sie ruhten. « Michel de Montaigne, Essais. Leipzig 1967, S. 309. (Von der Erfahrung).

38 Vgl. dazu Alain Supiot, Homo juridicus, S. 125. Supiot verweist dort auf den scholastischen Rechtsgelehrten Accursius, der die Frage, ob Juristen auch Theologie studieren sollten, verneinend antwortete: »Alles lässt sich im Körper des Rechts finden«. 
konstituierenden Wertesystems erhebt. ${ }^{39}$ Dieser Negativität im Kern des Rechts, die gegenwärtig durch den rechtspositivistischen Ansatz verdrängt wird, versuchen Legendre und Supiot durch den Terminus des Dogmatischen neue Geltung zu verschaffen:

Das griechische dogma verweist auf etwas, das scheint, erscheint, sich zu sehen gibt, bis hin zur Täuschung. Wenn man diesen Begriff mit der doxa in Verbindung setzt [...], führt es uns in zwei Bedeutungsdimensionen, die die Systeme zur sozialen Organisation des Diskurses mobilisieren: einerseits die Gründungsaxiome, Prinzipien oder Beschlüsse; andererseits die Ehrungen, die Beschönigung und das Dekor. ${ }^{40}$

Das Dogma verweist somit auf genau jenen existenziellen Widerspruch, der in der konstitutiven Leere der menschlichen Existenz seinen Ursprung findet - jener Leere, die für die menschliche Existenz so lebensnotwendig wie unerträglich ist, und die deshalb aufrechterhalten und zugleich verschleiert werden muss. Das Projekt der Kultur, so könnte man es formulieren, besteht somit darin, die unaussprechliche Leere durch Metaphorisierung, d.h. durch ein adäquates In-Worte-Fassen zu bezähmen und zu domestizieren. ${ }^{41}$

$I V$. Mit Blick auf die Aufgabe der Metaphorisierung der Negativität fällt nun den Institutionen eine entscheidende Funktion zu. Sie müssen die »Repräsentation durch etwas leisten«, wie Anton Schütz betont, »[n]ichts ist nicht genug.« Was dann aber konkret verarbeitet wird, ist von untergeordneter Bedeutung: »Ein aus Fälschungen gepatchworkter Verschlag, der die Subjektivität von ihrem Abgrund trennt, ist genug. $\aleph^{42}$ Es geht darum, den »menschlichen Abgrund« erträglich zu gestalten und der Alteritätsdimension der individuellen und kulturellen Identitätskonstitution ihre Legitimation zu verleihen.

Über die Einschaltung der Institutionalität obliegt es somit einer jeden Gesellschaft, auf emblematische Weise eine fiktive Entität, eine Referenz, zu errichten, die die Leere besetzt und als sicht- und sprechbarer Garant die Identität der Kultur und ihrer Subjekte authentifiziert. Mit Legendre gesprochen geht es darum, den leeren Platz als bevölkerten Ort zu inszenieren, als Schirm, auf den sich das System der gesellschaftlichen Objekte in ihrer Eigenschaft als sprachliche Konstruktionen stützt. Die emblematische Funktion besteht dann darin, diesen Platz zu bezeichnen - »Da ist er! « - und in ihn das Szenario einer kausalen Repräsentation einzuschreiben. ${ }^{43}$

Mit Hilfe jener Inszenierungsarbeit rufen die Institutionen somit den notwendigen metaphysischen Dritten ins Leben, um in seinem Namen zu sprechen und zu handeln. Die Institutionalität des menschlichen Lebens bezeichnet Legendre entsprechend als jene Dimension, die »als fiktionale Ordnung [...] dazu bestimmt ist, das Unsprechbare

39 Alain Supiot, Homo juridicus, S. 124.

40 Pierre Legendre, Anthropologie dogmatique. Définition d'un concept. In: École pratique des hautes études, Section des sciences religieuses. Annuaire. Tome 105, 1996-1997, 1996, S. 23-43.

41 Vgl. Georg Mein, Choreografien des Selbst, S. 26.

42 Anton Schütz, »Die Institution erhören. Echographik des gewöhnlichen Fanatismus.« In: Rechtshistorisches Journal 17/1998, S. 311-333, hier: S. 327.

43 Pierre Legendre, Über die Gesellschaft als Text. Grundzüge einer dogmatischen Anthropologie. Aus dem Französischen von Sabine Hackbarth. Wien, Berlin, 2012, S. 107. 
in Szene zu setzen und, ausgehend von dieser Gründungsrepräsentation, die normativen Wirkungen zu zeitigen, die wir, die Bewohner des Abendlandes, das Recht nennen. $\aleph^{44}$

Eine Gesellschaft muss begründet sein, um zu sprechen, d.h. sie muss befugt sein, einen Diskurs zu halten, der der Gesellschaft als solcher zuzurechnen ist und nicht den einzelnen Individuen. Ein solches fiktives Subjekt zu errichten, ist das primäre Ziel der institutionellen Montagen. Sobald dieser logische Schritt getan ist, ergibt sich daraus das Recht wie eine Folge, als Diskurs, der auf das monumentale Subjekt zurückgeführt werden kann. ${ }^{45}$

Indem sie so im Sinne konzeptueller Rahmungen sprachlich und ästhetisch die Referenz, d.h. eben jene notwendigen, für die Reproduktion der Menschheit unabdingbaren, Fiktionen inszenieren, ${ }^{46}$ kreieren die Institutionen eine kulturspezifische Sprach- und Bildordnung, die zum Ursprung der Kultur und des Rechts wird. Durch das Schaffen der Illusion einer Referenzialität von Sprache, Recht und Kultur authentifizieren sie das normative Fundament der Identitätsdimension, sie werden zum »Begründer der Montagen der Normativität in jeder Gesellschaft. « ${ }^{47}$

Die römische Formel Vitam instituere hat insofern nichts von ihrer Bedeutung eingebüßt. Institutionen richten das Leben ein, weil sie die symbolische Ordnung der Kultur nicht nur vermitteln, sondern auch garantieren. Sie antizipieren die subjektive Konstruktion des Individuums, in dem sie ihm ab initio den Status einer Person, persona, verleihen; ${ }^{48}$ sie lehren das neu ankommende Subjekt, sich mit den von der kulturellen Ordnung fabrizierten Bildern zu identifizieren. Legendre hebt in seiner Dogmatischen Anthropologie genau diese Dimension des Institutionellen immer wieder hervor. Institutionen sind in seiner Perspektive - sehr vereinfacht ausgedrückt $->$ Bildgebungsmaschinen`, die das Subjekt mit der kulturell fabrizierten Ordnung und damit mit der Idee der Menschheit selbst korrelieren:

Indem er sich im anderen seines Selbst erkennt, begegnet der Mensch dem Menschen, der Menschheit in sich. Er wird mit der Menschheit identisch und die Menschheit mit der Welt, mit dem Universum der Alterität. Das einzelne Individuum ist die Menschheit, es ist die Welt. ${ }^{49}$

Legendre mangelt es nicht an Deutlichkeit, wenn er betont: »In einem institutionellen System impliziert die Logik der Repräsentation das, was ich die Gefangennahme des Subjekts nenne. $\ll^{50}$ Mit anderen Worten, für Legendre gibt es kein sprechendes Subjekt »unabhängig von einem kulturell jeweils variabel institutionalisierten Normensystem, in dem Individuen humanisiert und dem sie verbindlich eingezeichnet werden. ${ }^{51}$

44 Pierre Legendre, Das politische Begehren Gottes, S. 133.

45 Ebd., S.190.

46 Vgl. Georg Mein, Choreografien des Selbst, S. 31.

47 Pierre Legendre, Das politische Begehren Gottes, S. 245.

48 Vgl. Péruisset-Fache, L'éducation d'homo sapiens, S.31 f.

49 Pierre Legendre, Gott im Spiegel, S. 242.

50 Pierre Legendre, Das politische Begehren Gottes, S. 512.

51 Clemens Pornschlegel: »Man muss es nicht für wahr halten, man muss es nur für notwendig halten.« Zur Figur des Gesetzgebers bei Pierre Legendre. In: Georg Mein (Hg.), Die Zivilisation des Interpreten, S. 243-257, hier S. 245. 
Das Paradoxe daran ist, dass die Institutionen ihre Arbeit mehr oder minder unbemerkt, gleichsam hinter dem Rücken der Subjekte vollziehen. Die instituierten Subjekte interpretieren das Gesetz, dem sie unterliegen, nicht als ein externes, von außen auferlegtes, sondern als selbstgegebenes und damit Autonomie verheißendes Gebot der eigenen Vernunft. Auf diese Weise wird das Paradox einer Freiheit durch Unterwerfung lebbar und das autonome Subjekt erscheint als ein Wunder der Dressur. Anders formuliert, die Macht der Institution in der Moderne »steht und fällt mit dem Gelingen der modernen Scheingeschäfte Verantwortungstransfer und Institutionstransfer, also der rechtswirksamen Übernahme der Institutionslast durch das instituierte Subjekt. $\ll^{52}$

$V$. Institutionen richten das Leben ein, indem sie das Subjekt einer von ihnen errichteten Normativität einschreiben. Der Grund, auf dem sie ruhen, ist allerdings leer; Institutionen sind daher immer auch ein Trick, eine Inszenierung, durch die das Subjekt vor dem Abgrund des Nichts abgeschirmt wird. Zu unterscheiden ist daher die leere Kategorie der Referenz von ihren jeweiligen Fülldiskursen (theologisch, metaphysisch oder säkular), die als unabdingbare Fiktionen für die Reproduktion des Menschen unverzichtbar sind. Denn in ihrer rohen Natürlichkeit ist die Welt für den Menschen überhaupt nicht zugänglich; erfassbar wird Wirklichkeit allein in inszenierter Form, d.h. durch Zeichen vermittelt, durch Worte repräsentiert, durch Kunst theatralisiert. Es geht um die Einsicht, in Legendres Worten, dass der Mensch keinen direkten, also rein animalischen oder sensorischen Zugang zu den Objekten der Welt hat. Seine Verbindung zu ihr setzt eine Leere voraus, das Funktionieren einer leeren Bühne, die von Worten bevölkert ist und die wir mit Recht (und mit Bezug zum Kino) als Leinwand der Worte bezeichnen können. Die Welt wird Repräsentation. ${ }^{53}$

Schon Nietzsche wusste, dass der Mensch nur in einem von Mythen umstellten Horizont leben kann, da erst durch die überhistorische Macht des mythologischen Wissens der Blick des Menschen von seinem konkreten Sein abgelenkt wird, »hin zu dem, was dem Dasein den Charakter des Ewigen und Gleichbedeutenden gibt «. ${ }^{54}$ Erst durch diese Inszenierungstechniken wird es möglich, den Abgrund zu bewohnen; sie sind elementarer Bestandteil des Programms, das jede Gesellschaft, europäisch oder asiatisch, antik oder industrialisiert, notwendig realisieren muss: des Programms, das Subjekt zur Welt kommen zu lassen. Erst durch den interpretierenden Zugriff mit Hilfe symbolischer und ästhetischer Formen schafft sich das Subjekt seine Wahrheit und damit eine Welt, die es bewohnen kann. Dabei ist dieses Unterfangen so unverzichtbar wie tragisch. Erich Rothacker hat das Denkbild geprägt, demzufolge die Menschen als »symbolische Vögel« auf dem Rücken eines leviathanischen Seeungeheuers verzweifelt versuchen, ihre Nester einzurichten:

In ungeheuren Wogen umspült eine völlig unfaßliche Wirklichkeit uns endliche Wesen. An sich von erhabener Gleichgültigkeit gegenüber unseren Sorgen, Leiden, Freuden. Inmitten dieses Ungeheuerlichen richtet sich die Menschheit ein. Wie eine

52 Anton Schütz, Die Institution erhören, S. 322.

53 Pierre Legendre, Vom Imperativ der Interpretation, S. 16.

54 Friedrich Nietzsche, Unzeitgemäße Betrachtungen II. In: Sämtliche Werke. Kritische Studienausgabe in 15 Bdn. hrsg. von Giorgio Colli und Mazzino Montinari, München 1988, Bd. I, S. 330 . 
Vogelfamilie, die auf dem ihr unverständlichen Rücken eines auf der Meeresoberfläche schwimmenden Seeungeheuers nistet, ohne zu ahnen, daß dieser Riesenwal auch einmal tauchen könnte. Sie nistet mit Behagen, wenn sie auf dem Rücken Boden findet und unterschlüpfen kann. Trifft sie dazwischen eine Meereswoge, so sinkt sie in das Grab, aber andere nisten weiter in Freude und Leid. Diese symbolischen Vögel richten sich so ein, so gut es geht, und erdeuten sich den Rücken des Seeungeheuers, so weit es ihnen gelingt, als ihre Welt. ${ }^{55}$

Im Rahmen dieses Unterfangens des \Erdeutens kommt nun die Kunst ins Spiel, von der Legendre behauptet, sie sei ein »metaphysischer Bluff«, da sie nicht die Welt entziffert, »sondern das, was hinter dem Sichtbaren liegt, etwas Formloses, dem sie eine Form geben soll. « ${ }^{56}$ Mit anderen Worten, erst der ästhetische Zugriff macht aus der rohen Natur eine Wirklichkeit für den Menschen. Welt lässt sich nur dann verstehend deuten, wenn sie in Narrationen, Mythen und Bilder kontextualisiert und damit als geformte Wirklichkeit vor Augen steht. Sinn ist nichts, was der Materie selbst entspringt, sondern stets das Produkt menschlichen Machens. Immanuel Kant hat dies in seiner Kritik der Urteilskraft mit Blick auf die Struktur des ästhetischen Urteils als »zweckfreie Zweckmäßigkeit« beschrieben: Der Mensch unterstellt der Wirklichkeit einen telos, da er sie nur so sinnhaft erfassen kann. Kant verfolgt hier einen Gedanken weiter, den er bereits in der Kritik der reinen Vernunft entwickelt hat. Dort argumentiert er, dass es mit Blick auf das spekulative Interesse der Vernunft schlichtweg notwendig sei, »alle Anordnung in der Welt so anzusehen, als ob sie aus Absicht einer allerhöchsten Vernunft entsprossen wäre. ${ }^{57}$ Das Entscheidende in diesem Zitat ist das als $o b$, durch das der Natur zumindest hypothetisch ein Telos unterstellt wird. Und eben dieser Gedanke wird in der Kritik der Urteilskraft wieder aufgegriffen und zu einem Prinzip a priori der reflektierenden Urteilskraft ausgebaut. Allerdings gilt dieses Prinzip eben nur »in subjektiver Rücksicht «, ${ }^{58}$ so Kant, denn es schreibt nicht der Natur (als Autonomie) ein Gesetz vor, sondern reglementiert das Reflexionsverfahren der Urteilskraft selbst. Mit anderen Worten, auch wenn die reflektierende Urteilskraft aus der Natur selbst kein apriorisches Gesetz ihrer Ordnungsmechanismen ableiten kann, wird doch eine für den Verstand erkennbare Ordnung angenommen bzw. unterstellt. Der ästhetische Blick auf die Welt lässt selbige für den Menschen als sinnvoll erscheinen - und das ist der entscheidende Punkt, um den es auch Legendre geht, wenn er der Kunst jenen zentralen Stellenwert zuspricht.

Wenn Kunst bzw. die ästhetische Dimension an sich in diesem Sinne konstitutiver Bestandteil des Kulturellen ist, weil ohne sie die institutionelle Dimension überhaupt nicht denkbar ist, dann stellt sich abschließend die Frage nach der ästhetischen Dimension des Rechts.

Wir hatten gesagt, dass Institutionen der Ort sind, an dem die Referenz ihre Stimme und ihre Gestalt erhält. D.h. Institutionen inszenieren eine Fiktion, die den menschlichen

55 Erich Rothacker, Zur Genealogie des menschlichen Bewusstseins. Bonn 1966, S. 211.

56 Pierre Legendre, Der dogmatische Wert des Ästhetischen. In: Zeitschrift für interkulturelle Germanistik 3 (2012) H.1, S. 163-172, hier S. 170.

57 Immanuel Kant, Kritik der reinen Vernunft. In: Werkausgabe, Bd. III-IV, B 714.

58 Immanuel Kant, Kritik der Urteilskraft (KdU). Werkausgabe, hrsg. von Wilhelm Weischedel. Frankfurt a.M.1995, Bd. X, B VII. 
Abgrund, d.h. seine rohe Natürlichkeit mit einer kulturspezifischen Sprach- und Bildordnung füllt, auf die sich die kulturelle und subjektive Identität in ihrer Alterität konstituierend beziehen kann. Um es, mit Legendre, sehr plastisch zu formulieren: »[E]s genügt nicht, Menschenfleisch herzustellen, der Mensch braucht einen Grund zu leben. « ${ }^{59}$ Als 〉Sprachrohr« und 〉Bildgebungsmaschinen` der Referenz umstellen Institutionen sprachlich und piktural jenen Ort der Leere, »an dem die Verhältnisse der Macht und der Wahrheit gesponnen « werden, ${ }^{60}$ und begründen so die Normativität - also das Recht. Im Kern geht es darum, dass sich das Recht im wahrsten Sinne des Wortes ästhetisch in Szene setzen muss, um so sichtbar zu machen, »was für den Menschen auf seiner subjektiven Reise zwischen Geburt und Tod Gesetz ist. $«{ }^{61}$ Diese ästhetische Dimension des Rechts lässt sich vor allem mit dem Blick auf den Begriff des ius personam veranschaulichen, welchen Legendre mit Blick auf die Etymologie des Begriffs persona als »Recht der Masken « übersetzt. ${ }^{62}$ Kultur ist gleichsam der Ort einer Masken-Ausgabe, durch deren Überstülpen das Individuum an das Gesetz gebunden wird. ${ }^{63}$ Seit der spezifischen Liaison von Christentum und Römischen Recht verwaltet in der abendländischen Tradition das Recht die Masken. Das Recht handhabt die »genealogischen Bilder « des Ursprungs ${ }^{64}$ und ermöglicht es dem Individuum so, im Spiegel des Rechts ein Subjekt, d.h. eine Maske zu werden, den Status eines Bildes in einer kulturell spezifizierten - referenzierten - Bildordnung anzunehmen. ${ }^{65}$

Diese spezifische Leistung des abendländischen Rechts ist ohne die Dimension der Ästhetik nicht denkbar. Dementsprechend erklärt Legendre die Kunst des Rechts im Sinne der Definition in den Digesten ius est ars boni et aequi ${ }^{66}$ zur Kunst im eigentlichen Sinne. Aufgabe dieser Rechtskunst ist Peter Goodrich zufolge die »Konstruktion eines Spiegelbilds, eines Porträts oder einer Ikone, die das Antlitz des Rechts in menschlicher Gestalt repräsentiert. « ${ }^{67}$ In der 1531 von Alciato begründeten Tradition der juristischen Embleme fand diese Funktion des Rechts ihren expliziten Ausdruck. In der Handhabung emblematischer Darstellungen des Rechts übernahmen die Juristen die Aufgabe, die Macht sichtbar zu machen, um so zugängliche Exemplare rechtmäßiger Gesinnungen und Verhaltensweisen zur Verfügung zu stellen. ${ }^{68} \mathrm{Ganz}$ im Sinne der repräsentationslogischen Konzeption des Menschen als bildgebundenes Kulturwesen gingen die Emblematiker davon aus, dass die Einschreibung von Moral, Recht und Norm in die Seele

59 Pierre Legendre, Vom Imperativ der Interpretation, S. 69.

60 Pierre Legendre, Das politische Begehren Gottes, S. 147.

61 Pierre Legendre, Der dogmatische Wert des Ästhetischen, S. $163 \mathrm{f}$.

62 Pierre Legendre, Das politische Begehren Gottes, S. 294.

63 Vgl. Peter Goodrich, Languages of Law. From Logics of Memory to Nomadic Masks. London 1990, S. 263.

64 Pierre Legendre, Gott im Spiegel, S. 334.

65 Goodrich, Languages of Law, S. 290.

66 Pierre Legendre, Das politische Begehren Gottes, S.133, mit Verweis auf Digestum 1,1,1.

67 Vgl. Goodrich, Languages of Law, S. 289.

$68 \mathrm{Vgl}$. Goodrich, Legal Emblems and the Art of Law. Obiter depicta as the Vision of Governance, Cambridge 2013, S. vx. 
des Menschen über das Auge, d.h. über den Einsatz der Bilder, im Sinne von imagines agentes, laufen müsse. ${ }^{69}$

In ihrer Dreigliedrigkeit, aus inscriptio, pictura und subscriptio, trägt die Rechtsemblematik dabei nicht nur der Trinität, d.h. der christlichen Tradition des dualistisch (politisch/theologisch) strukturierten Rechts Rechnung, ${ }^{70}$ sondern spiegelt zudem die trianguläre Kernstruktur der menschlichen Existenz wider, in der sich »Bild, Körper [und] Wort ${ }^{71}$ um einen Ort der Leere gruppieren und diesen verstellen. Als ästhetisch greifbare Verkörperung der normativen Relation zwischen Wort und Bild mobilisiert das Emblem den Blick des Subjekts, das in ihm den Grund des Gesetzes und durch seine Verinnerlichung letztlich sich selbst als authentifiziertes Bild in einer institutionell legitimierten Sinnordnung erkennen kann. Dem Subjekt wird der »nicht juridische[] Schauplatz des Rechts « eingelagert ${ }^{72}$ - ein Vorgang, der nicht nur für die Konstitution des institutionalisierten Subjekts unabdingbar ist, sondern ebenso für die Wirksamkeit des Rechts selbst, das ohne den Prozess der Anbindung, ohne den anerkennenden Blick des Subjekts, seinen Sinn, seine Wirksamkeit verliert. »Wir befinden uns im Spiel des Spiegels, durch das - wie in der scholastischen Montage der Imago Dei et Mundi - Gott von der Welt sein eigenes Bild empfängt. « ${ }^{73}$

In der Emblematik wird deutlich, dass auch die Zusammenschau von Recht und Kultur gewissermaßen als ein institutionell vermitteltes Spiegelverhältnis zu begreifen ist. Durch das Einbeziehen der ästhetischen Dimension - die im Rahmen der Tradition der juristischen Emblematik noch explizit präsent war, heute aber lediglich als ein mediales Randphänomen im Feld des Juridischen angesehen wird -, wird das Recht als der zentrale Vermittler eines fiktiven Fundaments der Macht erkennbar, das den Kern der kulturellen Identität bildet. Im Spiegel des Rechts konstituiert sich das Bild des kulturellen Ichs, welches der Institutionen bedarf, um wie ein menschlicher Körper sprechen und handeln zu können. Nur als ein solcher Körper kann die Kultur als >monumentales Subjekt` auftreten und so als rechtmäßiger Garant den Blick seiner Subjekte in den kulturellen Spiegel authentifizieren.

69 Matthaeus Merian, Emblematum ethico-politicorum centuria (Köln: Joharin de Bry, 1619), Kommentar zum Emblem 88 (Oculata fides), zitiert nach Peter Goodrich, Legal Emblems and the Art of Law, S. xvii.

70 Vgl. ebd.

71 Pierre Legendre, Das politische Begehren Gottes, S. 61.

72 Vgl. Pierre Legendre, Über die Gesellschaft als Text, S. 103.

73 Pierre Legendre, Das politische Begehren Gottes, S. 342. 